\title{
The short-term effects of antenna insulation thickness on path losses in wireless telemetry implants at microwave frequencies
}

\author{
Lukas Kneisz, Michael Schermann, Ewald Unger, Michael Haller, Matthias Krenn, \\ Winfried Mayr
}

Center for Medical Physics and Biomedical Engineering, Medical University of Vienna, Austria

\begin{abstract}
Various physiological parameters can be monitored non-invasively using wireless biotelemetry links. The development of sophisticated ultra low power consuming transceivers allows the transmission of large amounts of data from the inside of the body to an external receiver in real time at microwave frequencies.Antenna impedance matching is crucial for obtaining an acceptable propagation link budget in a wireless telemetry link. The dielectric properties of biological tissue induce detuning to transceiver antennas when implanted into the body. To counteract detuning problems, implant antennas are coated with biocompatible insulating material. The study investigates the propagation losses of a wireless communication link at different insulation thicknesses of medical grade silicone in the Industrial-Scientific-Medical (ISM) radio band at $2.45 \mathrm{GHz}$. The wireless link consisted of an implantable unit which was placed between two pads of tissue substitute material and an external receiver which was connected to a laptop. Predefined data packets were transmitted from the implant, the received packets were analyzed, packet errors and packet losses were logged and the received signal strength indicator values (RSSI) were recorded. Our results showed that the mean RSSI values of insulated transmitter antennas - embedded in tissue equivalent material - provide more safety distance to critical receiver sensitivity level than uncoated antennas. The conducted measurements let us conclude that with increasing thickness of the insulation layer, the antenna becomes less sensitive to detuning by adjacent tissue substitute material. Therefore tuned antennas are less influenced by the surrounding tissue after implantation. Key Words: biotelemetry, implantable antenna, path link losses
\end{abstract}

The transmission of physiological measurement data (e.g. glucose level, electrocardiogram, electromyogram, intracranial pressure [1]) from inside the human body to an external base station can either be done wired via a percutaneous lead or wireless via radiofrequency $(\mathrm{RF})$ transmission. Wireless telemetry offers the key advantages of improved patient comfort and minimized risk of infections. Furthermore measurement errors introduced by wire movement artifacts or even wire breakage are eliminated. RF transceivers are used to convert recorded biological signals in modulation of RF carrier signals and transmit this information via an antenna to an external device. Recent years developments in microsystems technology allow RF transceivers to become smaller in size, simultaneously increase performance and reduce power consumption. Communication distances of several meters can be covered; transfer rates up to the range of some Megabits per second (Mbps) can be achieved allowing signals with high-resolution to be transferred in real-time. Most of the conventional transceivers can be used bidirectional, offering the possibility for programming and adjusting implant parameters without surgical intervention and using bidirectional communication techniques to increase reliability of data transfer. The power present in a received radio signal is indicated as the so-called received signal strength indicator value (RSSI) and is influenced by the link losses in the system. These link losses include free space path losses, tissue absorption and losses due to antenna detuning. The latter can be minimized by performing measurements with a vector network analyzer (VNA) which characterizes the antenna in terms of reflection coefficient and resonance frequency. Reducing reflections between antenna and transceiver to a minimum and thus shifting the 


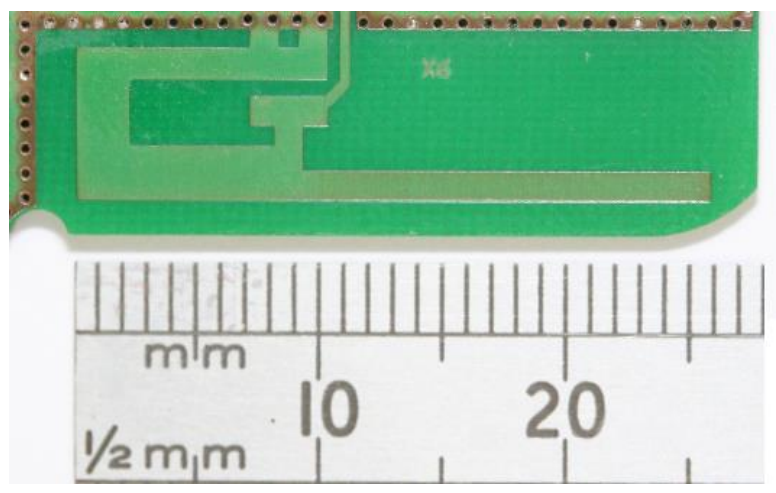

Fig 1. Photograph of printed inverted-F antenna (IFAO)

resonance frequency to the desired value is done by appropriate dimensioning of the antenna impedance network. The dielectric properties of biological tissue cause a previously matched antenna to detune when inserted into the tissue environment. As it is impossible to perform antenna measurements in vivo and correct for detuning, physical models are used to emulate a biological environment in terms of dielectric properties and propagation losses due to absorption of power. To predict the performance of an implant, numerous tissue-equivalent models exist, whereas some of them take into account the multilayered structures of different tissues [2].The aim of the present investigations was to determine the received signal strength and path loss while transmitting from a simulated implant via an antenna covered with an insulation of variable thickness through simulated biological tissue for various transmission distances. The overall aim is development of a reliable RF communication link with negligible dependence on tissue properties and anatomical variations.

\section{Materials and Methods}

Pilot experiments have been performed with different commercially available transceiver units which were equipped with planar inverted-F antennas (IFA, Figure 1) and an external base station with a meandered inverted-F antenna (MIFA). Both antenna types, MIFA

Table 1. Ingredients of tissue-equivalent pads (TP) in $\%$ by weight

\section{Ingredient}

Proportion [\%]

$\begin{array}{lc}\text { Deionized water } & 85.64 \\ \text { Agar } & 2.66 \\ \text { Sodium chloride } & 0.95 \\ \text { Sodium azide } & 0.05 \\ \text { TX-151 } & 2.14 \\ \text { Polyethylene powder } & 8.56\end{array}$

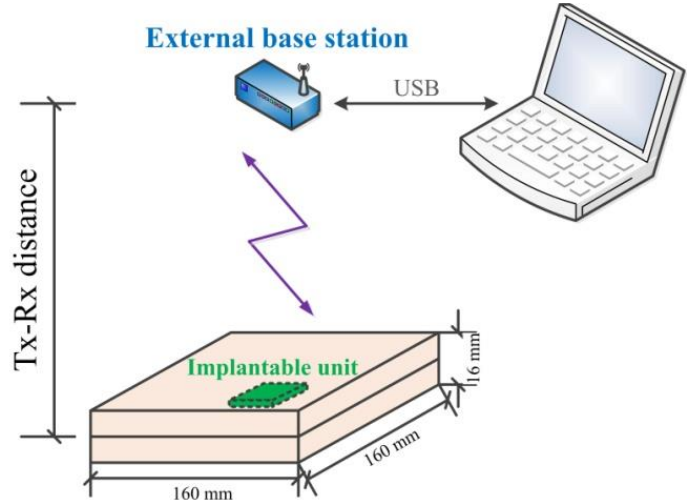

Fig 2. Experiment setup. Implantable unit is embedded in biological tissue-equivalent

and IFA, are quarter-wave monopoles on small ground planes. MIFA was not covered with any insulation material; whereas IFA was either uncoated (IFA0) or coated with medical grade silicone with layers of a thickness of $3 \mathrm{~mm}$ (IFA3) or of $6 \mathrm{~mm}$ (IFA6) of medical grade silicone. Prior to the experiments sub-miniature coaxial connectors were soldered to the printed circuit boards to connect the measurement system. Magnitudes of antenna input reflection coefficients (|S11|) were determined using an R\&S ZVL6 (Rohde\&Schwarz) calibrated vector network analyzer (Figure 3a). The impedance matching was done for each antenna separately. The communication link (Figure 2) consisted of a transmitter (Tx) placed within tissue phantom pads (TP) and an external base station acting as a receiver $(\mathrm{Rx})$ which was connected to a laptop and positioned at various distances from the transmitter. Biological tissue-equivalent phantom pads were constructed as proposed by Ito et al. [3]. The chemical composition is listed in Table 1 . The thickness was chosen to be $8 \mathrm{~mm}$ per pad, mimicking an implant site below the dermis and a fat layer. The implantable unit was programmed for data transmission with packets of 29 bytes payload and transmission intervals of $155 \mathrm{~ms}$. The average RSSI values, the packet error rate (PER) and packet lost rate (PLR) of the received packets were recorded. Measurements were performed at three different carrier frequencies (2402 MHz, $2446 \mathrm{MHz}$ and $2480 \mathrm{MHz}$ ) in the $2.45 \mathrm{GHz}$ ISM RF band. All measurements (IFA0, IFA3 and IFA6) were repeated with and without tissue

Table 2. Magnitude of S11 at the center frequency of $2.45 \mathrm{GHz}$ for the different insulation thicknesses

$\begin{array}{lccc}\mathrm{ft}=2.45 \mathrm{GHz} & \text { IFA0 } & \text { IFA3 } & \text { IFA6 } \\ \text { Free space (matched) } & -14.35 & -12.07 & -12.75 \\ \text { Tissue pad (detuned) } & -4.04 & -4.16 & -7.15\end{array}$




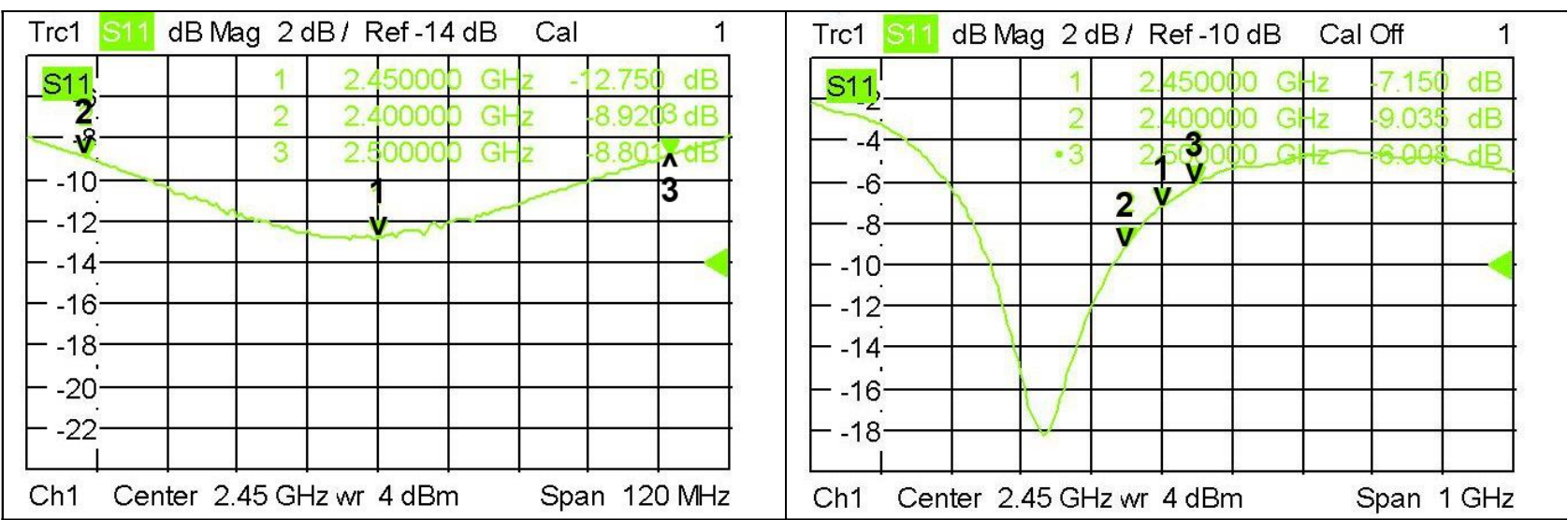

Fig 3. Simulated input $|S 11|$ of IFA6 matched in air (left panel) and detuning of the resonance frequency in biological tissue environment (right panel).

equivalent pads and referenced to free space (FS) transmission from uncovered antenna to uncovered antenna.

\section{Results and Discussion}

The output $|\mathrm{S} 11|$ of the VNA represents how much power transmitted by the transceiver is reflected from the antenna, hence the lower this value the more power is delivered to the antenna. Table 2 lists the magnitudes of $\mathrm{S} 11$ at the center frequency of $2.45 \mathrm{GHz}$ for the various insulation thicknesses. Detuning impact is lowest for IFA6 when placing the antenna in the tissueequivalent material (see Figure 3 for the graphical output of the VNA for IFA6). Figure 4 shows the recorded RSSI values for a transceiver output power of $4 \mathrm{dBm}(2.5 \mathrm{~mW})$. The receiver sensitivity of the CC2540 (Texas Instruments Inc, Dallas, TX) Systemon-Chip transceiver is $-93 \mathrm{dBm}$. Received packets with signal strengths below this value are not recognized and thus lost. A dashed indication line at this value is

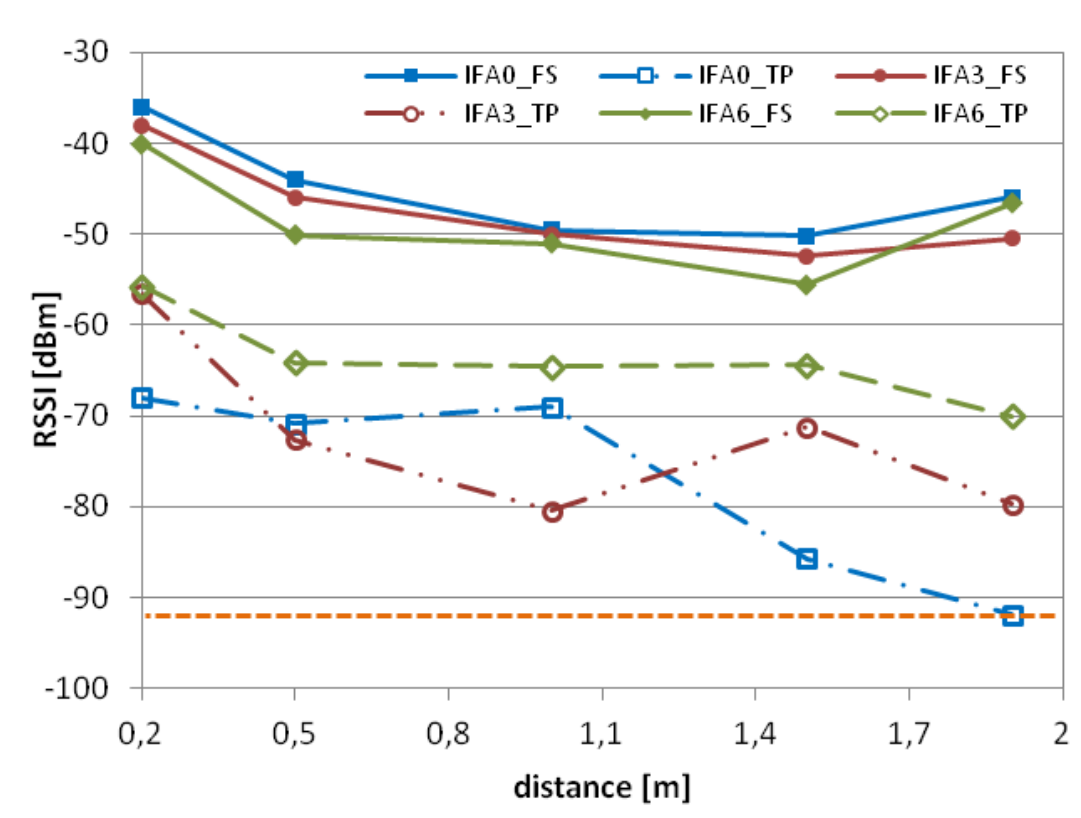

Fig 4. Received signal strength indicator values vs. distance for Pt $=4 \mathrm{dBm}$ at $\mathrm{ft}=2.446 \mathrm{GHz}$. The upper three curves reflect the behavior in free space, whereas the dashed curves depict the RSSI values at different distances with the tissue-equivalent pads. The lowest horizontal line marks the receiver sensitivity of the transceiver. 


\section{Effects of antenna insulation thickness on path losses in biotelemetry}

European Journal Translational Myology - Basic Applied Myology 2013; 23 (3): 91-94

depicted in Figure 4. The three upper solid curves depict the behavior of the RSSI values at free space conditions. We assume that the non-monotonic decrease with increasing distances was caused by interference. At a distance of 1.9 meters the sum of PER and PLR for IFA0 is $90 \%$ and thus no reliable communication is possible. The insulation covered antennas lead to total error rates of $1.7 \%$ for IFA3 and no errors for IFA6 at the same distance. Our experimental results showed that antenna insulation layers of $6 \mathrm{~mm}$ of medical grade silicone show great improvement of the total link budget of a wireless communication link through pads of tissue-equivalent material. However, constraints in implant dimensions may limit the maximum thickness of insulation layers. It must be noted, that the external antenna could be realized as a whip antenna. Power consumption and size are not crucial at the external base station and the typical efficiencies of whip antennas are higher than those of planar inverted-F antennas. Future investigations aim on long-term changes of the electrical properties of the insulation layer in chemical interaction with biological environment.

\section{Acknowledgements}

The authors gratefully acknowledge MED-EL (Innsbruck, Austria) for supporting this research work.

\section{Corresponding Author}

Lukas Kneisz, MSc, Center for Medical Physics and Biomedical Engineering, Medical University of Vienna, Waehringer Guertel 18-20 / AKH-4L 1090 Vienna, Austria. Phone +43 1404001990 e-mail: lukas.kneisz@meduniwien.ac.at

\section{References}

[1] Gemio J, Parron J, and Soler J. Human Body Effects on Implantable Antennas for ISM Bands Applications: Models Comparison and Propagation Losses Study. Prog Electromagn Res 2010; 110: 437-452.

[2] Ito K, Furuya K, Okano Y, Hamada L. Development and characteristics of a biological tissue-equivalent phantom for microwaves, Electronics and Communications 2001; vol. 84, no. 4: 66-77.

[3] Tofighi M R, Kawoos U, Neff S, Rosen A. Wireless intracranial pressure monitoring through scalp at microwave frequencies, Electron Lett 2006; vol. 42, no. 3: 148-150. 